\title{
AN APPLICATION OF $\lambda$-METHOD ON INEQUALITIES OF SHAFER-FINK'S TYPE
}

\author{
BRANKO J. MALEŠEVIĆ
}

Abstract. In this article $\lambda$-method of Mitrinović-Vasić [1] is applied to improve the upper bound for the arc sin function of L. Zhu [4].

Mathematics subject classification (2000): 26D05.

Key words and phrases: inverse sine, upper and lower bounds, Shafer-Fink type inequality.

\section{REFERENCES}

[1] D. S. Mitrinović, P. M. VAsić, Analytic inequalities. Springer-Verlag 1970.

[2] A. M. FINK, Two inequalities, Univ. Beograd. Publ. Elektrotehn. Fak., Ser. Mat., 6, (1995), 48-49. URL: (http://pefmath.etf.bg.ac.yu/)

[3] B. J. MALEŠEVIĆ, An application of $\lambda$-method on Shafer-Fink's inequality, Univ. Beograd. Publ. Elektrotehn. Fak., Ser. Mat., 8, (1997), 90-92. URL: (http://pefmath.etf.bg.ac.yu/)

[4] L. ZHU, On Shafer-Fink inequalities, Math. Inequal. Appl., 8, (4) (2005), 571-574. URL: (http: //www.mia-journal.com/)

[5] B. J. MALEŠEviĆ, One method for prooving inequalities by computer, Journal of Inequalities and Applications, 2007, Article ID 78691, 8 pages, 2007. doi:10.1155/2007/78691. URL: (http: //www.hindawi.com/journals/jia//)

[6] L. ZHU, A solution of a problem of Oppeheim, Math. Inequal. Appl., 10, (1) (2007), 57-61. URL: (http://www.mia-journal.com/)

[7] L. ZHU, On Shafer-Fink Type Inequality, Accepted in Journal of Inequalities and Applications, 2007, ID 67430. URL: (http://www.hindawi.com/journals/jia/) 